\title{
Clinical, laboratory, and radiological characteristics of COVID-19-infected children admitted to pediatric intensive care unit: a single-center experience
}

\author{
Heba Mostafa Ahmed ${ }^{1}$, Rehab Muhammad Abd El Kareem², Faten Mohamed Ali ${ }^{3}$, Ahmed Reda Sayed ${ }^{4}$ and
} Yasmen Awadalh Mohamed ${ }^{*}$

\begin{abstract}
Background: During the second wave of COVID-19, there is an increasing incidence of reported cases in children compared to the early wave. Data on the clinical and laboratory characteristics of COVID-19 in children are evolving, and reports on the characteristics and outcomes of severe COVID-19 in children are still under evaluation. We aimed to describe the clinical, laboratory, and radiological characteristics and outcomes of children with COVID-19 infection admitted to the pediatric intensive care unit (PICU).

Results: The study included 27 children with COVID-19 infection. Fever, respiratory, and gastrointestinal (GIT) symptoms were predominant presenting symptoms in our patients. The median age of our patients was 9 months ( $2 \mathrm{~m}-12$ years). Comorbidity was reported in 59.3\%. The typical laboratory findings were leukocytosis, lymphopenia, elevated C-reactive proteins levels, and elevated d-dimer levels. The most frequent radiological findings were groundglass opacities in $100 \%$ of patients and bilateral findings in $96 \%$, while cardiomegaly was found in $44 \%$ of patients. The multisystem inflammatory syndrome was reported in 33\% of patients with GIT symptoms were the most frequent presenting symptoms. Myocarditis was reported in $22 \%$ of patients. The mortality rate in this cohort was $14.8 \%$. On multivariate analysis, the only predictor of mortality was the development of MIS-C.

Conclusions: COVID-19 is more severe in children with comorbid conditions. Fever, respiratory and gastrointestinal (GIT) symptoms were predominant presenting symptoms. MIS-C is of increasing concern in children with high mortality rates.
\end{abstract}

Keywords: COVID-19, Pediatric intensive care unit, Multisystem inflammatory syndrome in children

\section{Background}

Coronaviruses are known pathogens in both animals and humans [1]. At the end of 2019, many cases of viral pneumonia were identified to be caused by a novel coronavirus in Wuhan city in China. The new virus was rapidly spreading, resulting in an epidemic all over China, and

\footnotetext{
*Correspondence: y.awadalh@yahoo.com

${ }^{1}$ Department of Pediatrics, Faculty of Medicine, Beni-Suef University, Beni Suef, Egypt

Full list of author information is available at the end of the article
}

then, many cases were reported in other countries all through the world. In February 2020, the World Health Organization (WHO) nominated the disease as coronavirus disease 2019 (COVID-19) [2], and on March 11, 2020, the WHO announced the COVID-19 as a worldwide pandemic [3]. Severe COVID -19 illness in adults is characterized by acute hypoxemia, multi-organ failure, and high mortality $[4,5]$. The risk factors for developing severe illness in adults include age, cardiac and respiratory comorbidities, obesity, lymphopenia, and high levels of D-dimer 
$[4,6]$. Initial reports from pediatric studies documented low infection rates and low incidence of pediatric intensive care unit (PICU) admissions [7, 8]. Early reports from China showed that critical illness was reported in only $0.6 \%$ of children with COVID-19 [8]. A large PICU report that included 48 children with COVID-19 admitted to 46 North American PICUs between March 14 and April 3, 2020, described these children's clinical characteristics and outcomes and reported a fatality rate of $4.2 \%$ [9]. Another large cohort included 632 children with proven COVID-19 infections all through the UK in the period from January 17 and July 3, 2020, showed that $18 \%$ (116 children) were admitted to critical care units [10], reflecting the increasing need for critical care management in children with COVID-19. This study aimed to prescribe the clinical characteristics and outcomes of children with COVID-19 admitted to PICU through the period from September 3, 2020, to December 31, 2020.

\section{Methods}

This was a prospective cross-sectional study. All children who presented to the emergency room with acute febrile illness or respiratory symptoms were subjected to full history taking, clinical examination, complete blood picture $(\mathrm{CBC}), \mathrm{C}$-reactive protein $(\mathrm{CRP})$, and computed tomography (CT) chest. Suspected COVID-19 infection was based on one of the following: the history of contact with COVID-19-confirmed case or history of contact with a patient with acute febrile illness from communities with confirmed cases, at least 2 of the following clinical features (fever and/or respiratory symptoms, lymphopenia in $\mathrm{CBC}$, characteristic CT findings), severe acute respiratory infection without an obvious cause. Suspected cases underwent either nasopharyngeal swab for PCR or antibody testing (anti-SARS-CV2 IgM and IgG). Positive critically ill patients were admitted to the COVID ward in the PICU, while stable cases were referred to an isolation hospital or home managed. Critically ill cases with negative PCR swap were isolated in the suspected COVID ward in the PICU. The test was repeated after $48 \mathrm{~h}$; if positive, transmitted to the COVID ward and if negative, transmitted to the non-COVID ward in the PICU. The criteria for PICU admission were: oxygen saturation $\leq 90 \%$ or $\mathrm{Pa} \mathrm{O} 2 / \mathrm{FiO} 2<300$ at room air, oxygen saturation $\leq 92 \%$ or $\mathrm{Pa} \mathrm{O} 2 / \mathrm{FiO} 2<200$ despite oxygen therapy, the presence of septic shock or hemodynamic instability not responding to fluid resuscitation with the need for inotrope support, patients with disturbed consciousness, and if respiratory symptoms were associated with other organs' failure. Diagnosis of the multisystem inflammatory syndrome in children (MIS-C) was based on the CDC case definition [11], including evidence of COVID19 infection (positive PCR or rapid test for COVID-19
IgG antibodies). The diagnosis of myocarditis was made based on the presence of left ventricular dysfunction by echocardiography and elevated serum troponin levels. Diagnosis of AKI was based on eGFR $<100 \mathrm{ml} /$ $\mathrm{min} / 1.73 \mathrm{~m} 2$. eGFR was calculated according to Schwartz formula $(\mathrm{eGFR}=\mathrm{kL} / \mathrm{Scre})$ where $\mathrm{k} 0.413, \mathrm{~L}=$ patient length or height, $\mathrm{Scr}=$ serum creatinine.

Patients were treated according to the Egyptian pediatric consensus recommendations [12] for COVID-19 infection, and MIS-C was treated according to the American Association of Pediatrics recommendations.[13]. For comparison, patients were divided into three groups according to age (age $<1$ year, age between $1-5$ years, age between $6-12$ years).

\subsection{PCR for COVID-19 detection}

The genesig ${ }^{\circledR}$ Real-Time PCR Coronavirus COVID-19 (CE IVD) is intended to be used to achieve qualitative detection of SARS-CoV-2 viral RNA extracted from nasopharyngeal swabs, oropharyngeal swabs, and sputum from patients. Soon after collection, nasopharyngeal and oropharyngeal secretions, each sample was inactivated by heating at $56 \mathrm{C}$ for $15 \mathrm{~min}$.

Extraction of SARS-CoV-2 viral RNA from nasopharyngeal swabs, oropharyngeal swabs, and sputum was done by Qiagen QIAamp DSP virus spin kit (Qiagen $\mathrm{GmbH}$, lot 163033809) on fully automated QIAcube (Qiagen, Germany). The RNA extraction sample volume of $700 \mu \mathrm{L}$ from the biological sample was used and resuspended with the COVID-19 primer/probe tube and the TM StepOne 2X RT-qPCR Master Mix then gently swirled to mix. The thermocycler was programmed with an initial cycle of $55^{\circ} \mathrm{C}$ for $10 \mathrm{~min}$, then $95^{\circ} \mathrm{C}$ for $2 \mathrm{~min}$ and 45 cycles of $95 \mathrm{C}$ for $10 \mathrm{~s}$ and $60 \mathrm{C}$ for $60 \mathrm{~s}$. Reverse transcription $10 \mathrm{~min} 55^{\circ} \mathrm{C}$ one cycle using TM StepOne (Applied Biosystem, Thermo Fisher Scientific).

\subsection{Detection of COVID-19 antibodies}

COVID-19 antibodies were detected using Elecsys AntiSARA-COV-2 commercial kits (Roche diagnostic GmbH, Germany) on Cobas e 411 (Roche, Hitachi, Germany).

\subsection{Measurement of D-dimer and CRP}

Both tests were measured using the nephelometric assay that utilizes antibody-coated latex particles. Both tests were done on MISPA_i 2, specific protein analyzer (AGAPPE Diagnostics Switzerland GmbH).

\subsection{Reporting of CT findings \\ 2.4.1 CORADS grading}

CORADS-1; COVID-19 is highly unremarkable, CT is normal or the findings seen are indicative of non-infectious disease, CORADS-2; suspicion level of COVID-19 
infection or the findings may suggest other infections; CORADS-3; indeterminate findings of COVID-19 infection, and the $\mathrm{Ct}$ findings include findings of infection and unsure if COVID-19 infection is present, CORAD-4; high suspicious for COVID-19 infection but not typical as being unilateral ground-glass opacity, confluent or being multifocal consolidative areas with atypical location, and CORADS-5; very high suspicion level of COVID-19 infection with typical CT findings [14].

\subsubsection{Semiquantitative scoring system}

The CT-SS was calculated based on the lobar involvement extent. Each of the five lung lobes was visually scored using a scale of $0-5$, with 0 indicates no remarkable involvement, 1 indicates less than 5\% lobar involvement, 2 indicates 5-25\% lobar involvement, 3 indicates 26-49\% lobar involvement, 4 indicates $50-75 \%$ lobar involvement, and 5 indicates more than $75 \%$ lobar involvement. The total CT score is calculated as the sum of the individual lobar scores ranging from 0 (no remarkable involvement) to 25 (maximum involvement). [15, 16 ] and defined as: 0 , none; 1 to 5 , minimal; 6 to 10 , mild; 11 to 15 , moderate; and $\geq 16$, severe involvement of the lungs [17].

\subsection{Statistical analysis}

The SPSS software (Statistical Package for the Social Sciences, version 22.0, SPSS Inc, Chicago, IL, USA) was used for analysis. Qualitative data were compared using the Chi-square test, and values were expressed as numbers and percentages. Parametric quantitative independent groups were examined using the $t$-test, or the Mann-Whitney test, and values were expressed as mean \pm standard deviation. One-way ANOVA test was used to compare continuous variables between the three age groups. Predictors for poor outcomes were studied using the binary logistic regression test. P-value was set at less than 0.05 for significant results.

\section{Results}

Out of 36 patients with suspected COVID-19 admitted to the PICU, 27 were proven positive for COVID19, including 16 (59\%) males and 11 (40.7\%) females. The median age of our study group was 9 months ( 2 months -12 years). The patients were categorized according to their ages into three groups: G1 age less than one year $(17 ; 63 \%), G 2: 1-5$ years $(3 ; 11 \%)$, and group $6-12$ years $(7 ; 26 \%)$. The median duration of pre-admission illness was four days (1-12 days). History of contact to COVID-19-positive patients was documented in 19 (70.4\%) patients. History of comorbid chronic disease was documented in 16 (59.3\%) of the patients (4 patients with congenital heart disease, one patient with bronchial asthma, 4 with central nervous system (CNS) disorders, 2 with chronic hematological disorders, 3 patients with nephrotic syndrome, one patient with growth hormone deficiency, and one patient with brain tumor). The presenting symptoms were fever (23; 85.2\%), cough 18 (66.7\%), shortness of breath $(15 ; 55.5 \%)$, gastrointestinal tract (GIT) symptoms (12; 44.4\%), convulsions(7;25.9\%), altered sensorium (11;59.3\%), shock (10;37\%). MIS-C was reported in 9 (33.3\%) of patients (Additional file 1: Digital Component 1). Nasal CPAP was indicated in 3 patients, while $6(22.2 \%)$ required invasive mechanical ventilation. The laboratory characteristics, complications, and medications received by the whole study group are shown in Table 1. High CRP $>5 \mathrm{mg} / \mathrm{dl}$ and ferritin levels $>200 \mathrm{mg} / \mathrm{dl}$ were detected in $100 \%$ of patients and elevated d-dimer more than $500 \mathrm{ng} / \mathrm{mL}$ in $63 \%$ of the patients. Anemia, leukocytosis, and lymphopenia were frequently observed. AKI was documented in 10 patients; 2 of them required renal replacement therapy. The radiological findings were ground-glass opacities in $49 \%$, bilateral findings in $30 \%$, cardiomegaly in $44 \%$. The median duration of PICU admission was 14 days (8-20 days). Echocardiographic findings included mild left ventricular dysfunction in 1 patient and moderate-to-severe left ventricular dysfunction in 5 patients; one of them had left coronary artery dilation and presented clinically with cardiogenic shock. As regarding treatments received by the patients, corticosteroids were given to 22 (81.5\%) patients. Eleven patients received pulse methylprednisolone (9 patients with MIS-C and 2 patients with myocarditis not fulfilling cafeteria for MIS-C), while 11 patients received oral prednisolone. IVIG was given to 3 patients with severe MIS-C, and one patient with severe COVID19 and shock. Low molecular weight heparin was given to 14 patients with high d-dimer, while antiplatelets were given to 17 patients. Comparison between the three age groups is shown in Table 2 with no significant differences between the three groups except for associated comorbidity, which was significantly higher in groups aged 1-12 than in patients younger than one year of age. At the same time, the frequency of cardiomegaly and myocarditis was significantly higher in patients younger than six years in comparison with older children. Comparisons between patients with and without MIS-C are shown in Table 3, Additional file 1: Digital Component 2. The frequency of GIT symptoms, shock at presentation, thrombocytopenia, lymphopenia, AKI, and elevated d-dimer levels were significantly higher in MIS-C patients. On logistic regression analysis, only AKI was an independent predictor for MIS-C (odds ratio; 2.35, SE; 1.14. 95\% CI; 1.12-98.92).

The mortality rate in our cohort was $14.8 \%$ (4 patients, 3 of them had MIS-C, and 3 had chronic comorbidity). 
Table 1 Clinical and laboratory characteristics of the study group (27 patients)

\begin{tabular}{|c|c|c|}
\hline & Frequency & Percent \\
\hline \multicolumn{3}{|l|}{ Laboratory data } \\
\hline \multicolumn{3}{|l|}{ CBC parameters } \\
\hline Anemia & 17 & 63.00 \\
\hline Thrombocytopenia $<150 \times 10^{3}$ & 6 & 22.20 \\
\hline Leukocytosis & 16 & 59.30 \\
\hline Lymphopenia < 1000/(cm ${ }^{3}$ & 14 & 51.90 \\
\hline Hypernatremia $>45 \mathrm{mEq} / \mathrm{L}$ & 14 & 51.90 \\
\hline \multicolumn{3}{|l|}{ K disturbances } \\
\hline Low & 6 & 22.20 \\
\hline High & 10 & 37.00 \\
\hline Metabolic acidosis (ph<7.2) & 12 & 44.40 \\
\hline High CRP $>5$ mg/dl & 27 & 100.00 \\
\hline High ferritin > 200 mg/dl) & 27 & 100.00 \\
\hline High d- dimer $>500$ mg/dl & 17 & 63.00 \\
\hline Coagulopathy (INR > 1.2) & 13 & 48.10 \\
\hline High troponin I (> 100 pg/ml) & 6 & 22.20 \\
\hline \multicolumn{3}{|l|}{ Complications } \\
\hline Pneumonia & 18 & 66.70 \\
\hline ARDS & 5 & 18.50 \\
\hline AKI $\left(e G F R<60 \mathrm{ml} / \mathrm{min} / \mathrm{m}^{2}\right)$ & 10 & 37.00 \\
\hline Myocarditis & 6 & 22.20 \\
\hline \multicolumn{3}{|l|}{ Medications } \\
\hline Vasoactive infusion & 8 & 29.60 \\
\hline Antibiotics & 26 & 96.30 \\
\hline Corticosteroids & 22 & 81.50 \\
\hline Anticoagulants & 14 & 51.80 \\
\hline Antiplatelets & 17 & 63.00 \\
\hline Remdesivir & 1 & 4.00 \\
\hline IVIG & 4 & 14.80 \\
\hline \multicolumn{3}{|l|}{ Outcomes } \\
\hline Discharge & 23 & 85.20 \\
\hline Mortality & 4 & 14.80 \\
\hline
\end{tabular}

AKI, acute kidney injury; ARDS, acute respiratory distress syndrome; $C B C$, complete blood counts; CRP, C-reactive protein; eGFR, estimated glomerular filtration rate; INR, international normalization ratio; IVIG, intravenous immunoglobulins; K, potassium

On logistic regression analysis, the development of MIS-C was a significant predictor of mortality (Table 4).

\section{Discussion}

Most of the data on critically ill COVID-19-infected children comes from developed countries; the clinical, laboratory, and radiological findings in children admitted to PICU with COVID-19 infection are described here for a better understanding of the underlying risk factors that can predict the outcome of children from a resourceconstrained developing country. Most of our patients were younger than one year of age in our cohort, while
Table 2 Comparison between the three age groups

\begin{tabular}{|c|c|c|c|c|}
\hline & \multicolumn{3}{|l|}{ Age group } & \multirow[t]{2}{*}{$\mathbf{p}$} \\
\hline & $<1$ y $(n=17)$ & $1-5$ y $(n=3)$ & $6-12$ y $(n=7)$ & \\
\hline \multicolumn{5}{|c|}{ Demographic and clinical characteristics } \\
\hline Male sex & $10(58.80 \%)$ & $2(66.70 \%)$ & $4(57.10 \%)$ & .960 \\
\hline $\begin{array}{l}\text { Contact to a posi- } \\
\text { tive case }\end{array}$ & $10(58.80 \%)$ & $2(66.70 \%)$ & $7(100 \%)$ & .132 \\
\hline Comorbidity & $7(41.20 \%)$ & $3(100 \%)$ & $6(85.70 \%)$ & .041 \\
\hline Fever & $13(76.50 \%)$ & $3(100 \%)$ & $7(100 \%)$ & .251 \\
\hline Cough & $7(41.20 \%)$ & $2(66.70 \%)$ & $5(71.40 \%)$ & .446 \\
\hline Dyspnea & $6(35.30 \%)$ & $3(100 \%)$ & $6(85.70 \%)$ & .677 \\
\hline GIT symptoms & $9(52.90 \%)$ & $1(33.30 \%)$ & $2(28.60 \%)$ & .506 \\
\hline $\mathrm{DCL}$ & $8(47.10 \%)$ & $2(66.70 \%)$ & $1(14.30 \%)$ & .207 \\
\hline Convulsions & $4(23.50 \%)$ & $2(66.70 \%)$ & $1(14.30 \%)$ & .208 \\
\hline Shock & $8(47.10 \%)$ & $1(33.30 \%)$ & $1(14.30 \%)$ & .316 \\
\hline \multicolumn{5}{|c|}{ Laboratory characteristics } \\
\hline Anemia & $11(64.70 \%)$ & $2(66.67 \%)$ & $4(57.14 \%)$ & .932 \\
\hline Thrombocytopenia & $5(29.4 \%)$ & $0(0.00 \%)$ & $1(14.3 \%)$ & .445 \\
\hline Leukocytosis & $8(47.1 \%)$ & $3(100 \%)$ & $5(71.4 \%)$ & .170 \\
\hline Hypernatremia & $8(47.1 \%)$ & $2(66.7 \%)$ & $4(57.1 \%)$ & .779 \\
\hline Hypokalemia & $4(23.5 \%)$ & $1(33.3 \%)$ & $1(14.3 \%)$ & .466 \\
\hline Hyperkalemia & $8(47.1 \%)$ & $0(0.00 \%)$ & $2(28.6 \%)$ & \\
\hline Hypoxemia & $7(41.2 \%)$ & $2(66.7 \%)$ & $3(42.9 \%)$ & .712 \\
\hline Coagulopathy & $7(41.2 \%)$ & $3(100 \%)$ & $3(42.9 \%)$ & .162 \\
\hline High d-dimer & $10(58.8 \%)$ & $3(100 \%)$ & $4(57.1 \%)$ & .369 \\
\hline High troponin I & $3(17.65 \%)$ & $1(33.33 \%$ & $2(28.6 \%)$ & .87 \\
\hline \multicolumn{5}{|l|}{ Complications } \\
\hline AKI & $7(41.2 \%)$ & $1(33.3 \%)$ & $2(28.6 \%)$ & .836 \\
\hline Myocarditis & $4(23.5 \%)$ & $1(33.3 \%)$ & $1(14.3 \%)$ & .020 \\
\hline Pneumonia & $8(47.1 \%)$ & $3(100 \%)$ & $7(100 \%)$ & .284 \\
\hline MIS-C & $5(29.4 \%)$ & $2(66.7 \%)$ & $2(28.6 \%)$ & .430 \\
\hline \multicolumn{5}{|l|}{ CT findings } \\
\hline \multicolumn{5}{|l|}{ CORADS } \\
\hline 3.00 & $4(23.5 \%)$ & $0(0.0 \%)$ & $0(0.0 \%)$ & .596 \\
\hline 4.00 & $9(52.9 \%)$ & $2(66.7 \%)$ & $5(71.4 \%)$ & \\
\hline 5.00 & $4(23.5 \%)$ & $1(33.3 \%)$ & $2(28.6 \%)$ & \\
\hline Pneumonia & 4 (23.5\%) & $2(66.7 \%)$ & $3(42.9 \%)$ & .284 \\
\hline Bilateral findings & $5(29.4 \%)$ & $1(33.3 \%)$ & $2(28.6 \%)$ & .227 \\
\hline Cardiomegaly & 11 (64.7\%) & $0(0.0 \%)$ & $1(14.3 \%)$ & .02 \\
\hline
\end{tabular}

AKI, acute kidney injury; GIT, gastrointestinal tract; MIS-c, multisystem inflammatory syndrome in children; CORADS, COVID-19 reporting and data system

only $11 \%$ of patients were in the group between 1 and 5 years of age. In line with our findings, Swann et al. [10] reported that the most common age group was younger than one year, and only 12.9 percent of their PICUadmitted patients were in the age group between 1 and 4 years. Comorbid conditions were reported in $59.3 \%$ of our patients; most of them were older than one year of age. Similar results were observed in the study by Swann 
Table 3 Comparison between patients with and without MIS-C

\begin{tabular}{lccl}
\hline & \multicolumn{2}{l}{ MIS-C } & p-value \\
\cline { 2 - 3 } & No & Yes & \\
\hline Clinical data & & & \\
Male sex & $11(61.1 \%)$ & $5(55.6 \%)$ & .78 \\
Comorbidity & $13(72.2 \%)$ & $3(33.3 \%)$ & .05 \\
GIT & $5(27.8 \%)$ & $7(77.8 \%)$ & .014 \\
Shock & $4(22.2 \%)$ & $6(66.7 \%)$ & .024 \\
Myocarditis & $2(11.1 \%)$ & $4(44.4 \%)$ & .05 \\
IMV & $2(11.1 \%)$ & $4(44.4 \%)$ & .05 \\
Mortality & $1(5.6 \%)$ & $3(33.3 \%)$ & .17 \\
Laboratory data & & & \\
Anemia & $10(55.6 \%$ & $7(77.8 \%)$ & .26 \\
Thrombocytopenia & $1(5.6 \%)$ & $5(55.6 \%)$ & .003 \\
Leukocytosis & $10(55.6 \%)$ & $6(66.7 \%)$ & .58 \\
Lymphopenia & $6(33.3 \%)$ & $8(88.9 \%)$ & .006 \\
AKI & $3(16.7 \%)$ & $7(77.8 \%)$ & .002 \\
Coagulopathy & $7(38.9 \%)$ & $6(66.7 \%)$ & .17 \\
High d-dimer & $8(44.4 \%)$ & $9(100.0 \%)$ & .005 \\
High troponin I & $2(11.1 \%)$ & $4(44.4 \%)$ & .91 \\
\hline AKI, & &
\end{tabular}

AKI, acute kidney injury; GIT, gastrointestinal tract; IMV, invasive mechanical ventilation; MIS-c, multisystem inflammatory syndrome

Table 4 Binary logistic regression analysis for predictors of mortality

\begin{tabular}{lllll}
\hline Variable & Odds ratio & SE & $\mathbf{9 5 \%} \mathbf{C l}$ & p-value \\
\hline Respiratory distress & 1.25 & 1.01 & $.17-9.02$ & .831 \\
GIT symptoms & .012 & 1.01 & $.11-5.77$ & .824 \\
Shock & 1.17 & 1.02 & $.16-8.52$ & .879 \\
AKI & 1.17 & 1.02 & $0.16-8.52$ & .879 \\
D-dimer $>1000 \mathrm{ng} / \mathrm{ml}$ & 1.6 & 1.23 & $.14-18.01$ & .751 \\
Myocarditis & .48 & .85 & $.09-2.55$ & .395 \\
MIS-C & 13.6 & 1.3 & $1.23-78.05$ & .034 \\
\hline
\end{tabular}

AKI, acute kidney injury; GIT, gastrointestinal tract; IMV, invasive mechanical ventilation; $\mathrm{K}$, potassium; MIS-c, multisystem inflammatory syndrome in children

et al. [10], who mentioned that $54.3 \%$ of their cohort had comorbidities. In comparison, Shekerdemian and her peers [9] reported a higher incidence of comorbidity among their patients (83\%), similar to that reported in adults [4-6]. Most of our cases had fever at presentation similar to what was reported in other studies $[9,10]$. In our cohort, fever and GIT symptoms were more prevalent in children younger than one year, while older age groups reported respiratory symptoms. In the study of Swann et al. [10], diarrhea and vomiting were reported in $34.5 \%$ and $39.7 \%$, respectively. Similarly, González-Dambrauskas et al. [18] reported that $35 \%$ of their patients presented GIT symptoms. Contrary to our results, in the study by Shekerdemian et al. [9], only one patient out of 48 presented GIT symptoms. The research time frames could still explain this discrepancy in the presenting symptoms. In contrast, the study with a higher incidence of respiratory symptoms was conducted out at an earlier time in 2020 year. Our study and the study by GonzálezDambrauskas et al. [18] were conducted during the 2nd half of 2020, indicating the changing nature of the virus during the first and second waves. In our study, IMV was indicated in $22.2 \%$ of patients. However, in the study by Shekerdemian et al. [9] and the study by GonzálezDambrauskas et al. [18], higher incidences of IMV were reported (38\% and 47\%, respectively). Compared to these two studies, the lower rate of IMV in our cohort may be due to the lower rates of severe respiratory symptoms and ARDS. In our study, 33\% of our patients met the CDC criteria of MIS-C, similar to what was reported by Swann et al. [10], where 32.7\% (38/116) of COVID19-positive children admitted to the PICU met the WHO criteria for MIS-C. In contrast to the study by Swann et al. [10], which reported higher rates of MIS-C in the older age group, there were no significant age differences in patients with and without MIS-C. Our explanation for this difference could be due to the different ethnic groups, including older children (up to 19 years of age). In patients with MIS-C, GIT symptoms predominated significantly compared to non-MIS-C patients, similar to those reported in other studies $[10,19]$. In addition, platelet counts and lymphocytic counts were significantly lower in MIS-C patients. Other researchers have reported similar findings $[10,19]$. Serum creatinine and d-dimer levels were also found to be significantly higher in patients with MIS-C. In the study by Swann et al. [10], higher creatinine levels were reported in patients with MIS-C. However, they did not comment on differences in d-dimer levels in both groups. Pereira et al. [20] reported that d-dimer levels of more than $1000 \mathrm{ng} / \mathrm{dl}$ were significantly associated with MIS-C (p-value; 007). In the current study, we reported a mortality rate of $14.8 \%$, which was higher than that reported in other studies. In the study conducted by Swann et al. [10], a 0.9\% (6/651) mortality rate was reported for a cohort of patients admitted to hospital and PICU wards. However, the mortality rate of PICU-admitted patients was not reported. Similarly, in the study of Shekerdemian et al. [9], the mortality rate was $4 \%$. However, at the time of submission, $19 \%$ of their cohort were still admitted with critical illnesses, so the total cohort's actual mortality could be understated. In our cohort, one of the four patients died had died only after $5 \mathrm{~h}$ of admission ( a case of nephrotic syndrome on treatment with mycophenolate mofetil complained of high-grade fever, abdominal pain, vomiting, diarrhea, and skin rash received home treatment without 
improvement of her complaints and presented to our ER after 8 days with circulatory shock and severe myocardial dysfunction necessitated IMV and inotropes support, but unfortunately she passed out and her swap revealed COVID-19 positive). Two of the remaining three patients had severe cerebral palsy and impaired respiratory function, while only one was previously healthy before admission. The presence of MIS-C was a significant predictor of mortality in this study. In the study by Pereira et al. [20], hypoxemia and MIS-C were associated with mortality. In our study, we found no association between the findings of the CT and the risk of MIS-C or mortality despite previous reports of this association [15, 17, 21]. This may be related to the predominance of GIT symptoms over respiratory symptoms, particularly among MIS-C patients. Although only one patient received antiviral therapy due to our limited resources, the outcome of our patients did not differ greatly from the other reports, as the most serious cases are those with MIS-C that may benefit from immunosuppressants than antiviral therapy.

Study strength: this study describes clinical, laboratory, and radiographic data of children admitted to PICU due to COVID-19 infection in a prospective study that is not limited to patient records and provides insight into differences in clinical settings of the disease and outcomes in a different ethnicity with limited resources.

Study limitations: small sample size as the disease is much milder in children than adults, and this study represents a single center's experience.

\section{Conclusions}

COVID-19 is more severe in children with comorbid conditions. Fever, respiratory, and gastrointestinal (GIT) symptoms were predominant presenting symptoms. MIS-C is of increasing concern in children with high mortality rates.

\begin{abstract}
Abbreviations
AKI: Acute kidney injury; CBC: Complete blood picture; CDC: Centers for Disease Control and Prevention; CRP: C-reactive protein; CT: Computed tomography; GIT: Gastrointestinal tract; MIS-C: Multisystem inflammatory syndrome in children; PICU: Pediatric intensive care unit; WHO: World Health Organization.
\end{abstract}

\section{Supplementary Information}

The online version contains supplementary material available at https://doi. org/10.1186/s43088-021-00168-x.

Additional file 1. Digital Component 1 and Digital Component 2.

\section{Acknowledgements}

We would like to acknowledge all nurses and pediatrics residents working at the PICU of our hospital for their tremendous efforts in taking care of the patients all the time without a rest.

\section{Authors' contributions}

HM analyzed and interpreted the patient data. RM \& FM supervised data collection and analysis. AR helped in data analysis. YA collected the data. All authors have read and approved the manuscript.

\section{Funding}

Not applicable.

\section{Availability of data and materials \\ Not applicable.}

\section{Declarations}

\section{Ethics approval and consent to participate}

This study was approved by the Ethics Committee of Beni-Suef University, Faculty of Medicine, and the ethics code was FWA00015574 FMBSUREC/2032021. Also, written informed consent was obtained from the parent of the participating children.

\section{Consent for publication \\ Not applicable.}

\section{Competing interests}

The authors declare that they have no conflict of interest.

\section{Author details}

${ }^{1}$ Department of Pediatrics, Faculty of Medicine, Beni-Suef University, Beni Suef, Egypt. ${ }^{2}$ Department of Clinical and Chemical Pathology, Faculty of Medicine, Beni-Suef University, Beni Suef, Egypt. ${ }^{3}$ Department of Radiodiagnosis, Faculty of Medicine, Beni-Suef University, Beni Suef, Egypt. ${ }^{4}$ Department of Medical Biochemistry and Molecular Biology, Faculty of Medicine, Beni-Suef University, Beni Suef, Egypt.

Received: 7 May 2021 Accepted: 2 November 2021

Published online: 14 November 2021

\section{References}

1. Weiss SR, Navas-Martin S (2005) Coronavirus pathogenesis and the emerging pathogen severe acute respiratory syndrome coronavirus. Microbiol Mol Biol Rev 69:635

2. World Health Organization (2020) Director-General's remarks at the media briefing on 2019-nCoV on 11 February 2020. http://www.who.int/ $\mathrm{dg} /$ speeches/detail/who-director-general-s-remarks-at-the media-briefing-on-2019-ncov-on-11-february-2020 (Accessed on February 12, 2020).

3. World Health Organization (WHO) (2020) WHO Director-General's opening remarks at the media briefing on COVID-19. Available at: https:// www.who.int/dg/speeches/detail/who-director-general-s-openingremarks-at-the-media-briefing-on-covid-19---11-march-2020

4. Richardson S, Hirsch JS, Narasimhan M, et al; and the Northwell COVID-19 Research Consortium (2020) Presenting characteristics, comorbidities, and outcomes among 5700 patients hospitalized with COVID-19 in the New York city area. [published correction appears in JAMA. 2020;323(20):2098]. JAMA: 323(20):2052-2059.

5. Bhatraju PK, Ghassemieh BJ, Nichols M et al (2020) Covid-19 in critically III patients in the Seattle region - case series. N Engl J Med 382(21):2012-2022

6. Chen R, Liang W, Jiang M, et al (2020) Medical Treatment Expert Group for COVID-19. Risk factors of fatal outcome in hospitalized subjects with coronavirus disease 2019 from a nationwide analysis in China [published online ahead of print April 15, 2020]. Chest. doi:https://doi.org/10.1016/j. chest.04.010

7. CDC COVID-19 Response Team (2020) Coronavirus disease 2019 in children - United States, February 12-April 2, 2020. MMWR Morb Mortal Wkly Rep 69(14):422-426

8. Dong Y, Mo X, Hu Y et al (2020) Epidemiology of COVID-19 among children in China. Pediatrics 145(6):1202

9. Shekerdemian LS, Mahmood NR, Wolfe KK et al (2020) Characteristics and outcomes of children with coronavirus disease 2019 (COVID-19) infection 
admitted to US and Canadian Pediatric Intensive Care Units. JAMA Pediatr 174(9):868-873

10. Swann OV, Holden KA, Lance T, Louisa P, Fairfield CJ, Drake TM et al (2020) Clinical characteristics of children and young people admitted to hospital with covid-19 in United Kingdom: prospective multicentre observational cohort study. BMJ 370:m3249

11. Centers for Disease Control and Prevention Health Alert Network (HAN) (2020) Multisystem Inflammatory Syndrome in Children (MIS-C) Associated with Coronavirus Disease 2019 (COVID-19). Available at: https:// emergency.cdc.gov/han/2020/han00432.asp

12. Mostafa AS, Abdalbaky A, Fouda EM et al (2020) Practical approach to COVID-19: an Egyptian pediatric consensus. Egypt Pediatric Assoc Gaz $68: 28$

13. AAP Interim Guidance on Multisystem Inflammatory Syndrome in Children (MIS-C). Available at: https://www.aap.org/en/pages/2019-novelcoronavirus-covid-19-infections/clinical-guidance/multisystem-infla mmatory-syndrome-in-children-mis-c-interim-guidance/ Accessed 14 Aug 2020.

14. COVID working group of the Dutch Radiological Society (2020) available at https://radiologyassistant.nl/chest/covid-19/corads-classification. Published 25/3/2020.

15. Francone M, lafrate F, Masci GM et al (2020) Chest CT score in COVID-19 patients: correlation with disease severity and short-term prognosis. EurRadiol 4:1-10
16. Pan F, Ye T, Sun P, Gui S, Liang B, Li L, Zheng D, Wang J, Hesketh RL, Yang L, Zheng C (2020) Time Course of Lung Changes at Chest CT during Recovery from Coronavirus Disease 2019 (COVID-19). Radiology 295:715-721

17. Hu Y, Zhan C, Chen C, Ai T, Xia L (2020) Chest CT findings related to mortality of patients with COVID-19: A retrospective case-series study. PLoS ONE 15(8):e0237302

18. González-Dambrauskas S, Vásquez-Hoyos P, Camporesi A et al (2020) Pediatric critical care and COVID-19. Pediatrics 146(3):e20201766

19. Whittaker E, Bamford A, Kenny J, et al (2020) PIMS-TS Study Group and EUCLIDS and PERFORM Consortia. Clinical characteristics of 58 children with a pediatric inflammatory multisystem syndrome temporally associated with SARS-CoV-2. JAMA. 21:324(3):259-269.

20. Pereira MFB, Litvinov N, Farhat SCL, Eisencraft AP, Gibelli MABC, Carvalho WB et al (2020) Severe clinical spectrum with high mortality in pediatric patients with COVID-19 and multisystem inflammatory syndrome. Clinics (Sao Paulo) 75:e2209

21. Hafez MAF (2020) The mean severity score and its correlation with common computed tomography chest manifestations in Egyptian patients with COVID-2019 pneumonia. Egypt J Radiol Nucl Med 51:254

\section{Publisher's Note}

Springer Nature remains neutral with regard to jurisdictional claims in published maps and institutional affiliations.

\section{Submit your manuscript to a SpringerOpen ${ }^{\circ}$ journal and benefit from:}

- Convenient online submission

$\checkmark$ Rigorous peer review

- Open access: articles freely available online

- High visibility within the field

- Retaining the copyright to your article

Submit your next manuscript at $\boldsymbol{\nabla}$ springeropen.com 